\title{
COMT Polymorphism and Memory Dedifferentiation in Old Age
}

\author{
Goran Papenberg \\ Max Planck Institute for Human Development and Karolinska \\ Institute \\ Irene E. Nagel \\ Freie Universität Berlin \\ Julia Schröder \\ Max Planck Institute for Molecular Genetics and Charité \\ Universitätsmedizin
}

Hauke R. Heekeren

Freie Universität Berlin

\author{
Lars Bäckman \\ Karolinska Institute
}

\author{
Wilfried Nietfeld \\ Max Planck Institute for Molecular Genetics \\ Lars Bertram \\ Max Planck Institute for Molecular Genetics \\ Ulman Lindenberger \\ Max Planck Institute for Human Development
}

\author{
Shu-Chen Li \\ Max Planck Institute for Human Development and TU Dresden
}

\begin{abstract}
According to a neurocomputational theory of cognitive aging, senescent changes in dopaminergic modulation lead to noisier and less differentiated processing. The authors tested a corollary hypothesis of this theory, according to which genetic predispositions of individual differences in prefrontal dopamine (DA) signaling may affect associations between memory functions, particularly in old age. Latent correlations between factors of verbal episodic memory and spatial working memory were compared between individuals carrying different allelic variants of the Catechol-O-Methyltransferase (COMT) Val158Met polymorphism, which influences DA availability in prefrontal cortex. In younger adults ( $n=973$ ), correlations between memory functions did not differ significantly among the 3 COMT genotypes $(r=.35)$; in older adults $(n=1333)$, however, the correlation was significantly higher in Val homozygotes $(r=.70)$, whose prefrontal DA availability is supposedly the lowest of all groups examined, than in heterozygotes and Met homozygotes (both $r \mathrm{~s}=.29$ ). Latent means of the episodic memory and working memory factors did not differ by COMT status within age groups. However, when restricting the analysis to the low-performing tertile of older adults $(n=443)$, we found that Val homozygotes showed lower levels of performance in both episodic memory and working memory than heterozygotes and Met homozygotes. In line with the neurocomputational theory, the observed dedifferentiation of memory functions in older Val homozygotes suggests that suboptimal dopaminergic modulation may underlie multiple facets of memory declines during aging. Future longitudinal work needs to test this conjecture more directly.
\end{abstract}

Keywords: COMT, dopamine, aging, dedifferentiation, memory

This article was published Online First July 8, 2013.

Goran Papenberg, Center for Lifespan Psychology, Max Planck Institute for Human Development, Berlin, Germany and Aging Research Center, Karolinska Institute, Stockholm, Sweden; Lars Bäckman, Aging Research Center, Karolinska Institute; Irene E. Nagel, Department of Education and Psychology, Freie Universität Berlin, Berlin, Germany; Wilfried Nietfeld, Max Planck Institute for Molecular Genetics, Berlin, Germany; Julia Schröder, Max Planck Institute for Molecular Genetics and Charité Universitätsmedizin, Berlin, Germany; Lars Bertram, Max Planck Institute for Molecular Genetics; Hauke R. Heekeren, Department of Education and Psychology, Freie Universität Berlin; Ulman Lindenberger, Center for Lifespan Psychology, Max Planck Institute for Human Development; and Shu-Chen Li, Center for Lifespan Psychology, Max Planck Institute for Human Development and Department of Psychology, Lifespan Developmental Neuroscience Section, TU Dresden, Dresden, Germany.
This research was supported by the Max Planck Society, including grants from the Innovation Fund of the Max Planck Society (M.FE.Abild0006). It was also supported by a grant from the German Federal Ministry of Education and Research to the Berlin Neurolmaging Center (01G00501). Lars Bäckman was supported by the Swedish Research Council, Swedish Brain Power, an Alexander von Humboldt Research Award, and by a donation from the af Jochnick Foundation. Goran Papenberg is a fellow of the International Max Planck Research School, The Life Course: Evolutionary and Ontogenetic Dynamics (LIFE). We thank Kirsten Becker, Ludmila Müller, Carolin Stockmeyer, and the other research assistants for their help.

Correspondence concerning this article should be addressed to Goran Papenberg, Aging Research Center, Karolinska Institute, Gävlegatan 16, SE-11330 Stockholm, Sweden. E-mail: goran.papenberg@ki.se or ShuChen Li, Department of Psychology, Lifespan Developmental Neuroscience Section, TU Dresden, Zellescher Weg 17, D-01062 Dresden, Germany. E-mail: shu-chen.li@tu-dresden.de 
The Catechol-O-Methyltransferase (COMT) gene codes for an enzyme that degrades dopamine (DA) in the prefrontal cortex, resulting in three to four times higher turnover rates in Val than in Met homozygotes (e.g., Lotta et al., 1995). Thus, Val homozygotes have lower levels of synaptic DA in prefrontal cortex than Met carriers. In light of DA's critical involvement in prefrontal functioning (e.g., Kimberg \& D’Esposito, 2003; Luciana, Depue, Arbisi, \& Leon, 1992; Sawaguchi \& Goldman-Rakic, 1991; Vijayraghavan, Wang, Birnbaum, Bruce, Williams, \& Arnsten, 2007; Williams \& Goldman-Rakic, 1995), studies have investigated the link between individual differences in the COMT gene and cognitive performance. Specifically, the Met allele has been associated with better performance in tasks assessing executive functioning (e.g., Nagel et al., 2008; Sheldrick et al., 2008) and working memory (e.g., Dumontheil et al., 2011; Nagel et al., 2008; Störmer, Passow, Biesenack, \& Li, 2012). However, several studies have failed to replicate these associations (e.g., Blanchard, Chamberlain, Roiser, Robbins, \& Müller, 2011; Bolton et al., 2010; de Frias et al., 2010; Zilles et al., 2012) and meta-analyses indicate that the COMT genotype has limited effects on cognitive functioning (Barnett, Jones, Robbins, \& Müller, 2007; Barnett, Scoriels, \& Munafò, 2008). These observations form part of a larger picture suggesting that individual genes exert small and often negligible effects on cognitive performance (Deary et al., 2010; Payton et al., 2009).

On the other hand, arguments have been made that brain processes are more likely to reveal influences of genetic polymorphisms, because they are more proximal to molecular mechanisms than the behaviors associated with them (Rasch, Papassotiropoulos, \& de Quervain, 2010). Brain imaging studies have shown that, in the absence of reliable behavioral differences between allelic groups during working memory tasks, Val homozygotes showed less focused neuronal activity in the cortical working memory network (Bertolino et al., 2006) and exhibited greater prefrontal brain activity (Egan et al., 2001), thus suggesting less efficient cognitive processing in carriers of this genotype. In addition to increased frontal activation, COMT Val homozygotes showed increased activation in the parietal lobes during a visuospatial working memory task (Dumontheil et al., 2011), decreased medial temporal lobe activity (Dennis et al., 2010), and increased prefrontal activity during episodic encoding (Dennis et al., 2010; Schott et al., 2006). Taken together, the extant evidence suggests that the Val allele appears to be associated with less efficient and less distinct recruitment of task-relevant brain networks (for review, see Witte \& Flöel, 2012).

Aging is associated with declines in the DA systems (for review, see Bäckman, Lindenberger, Li, \& Nyberg, 2010) and working memory depends critically on prefrontal DA (for review, see Cools \& D'Esposito, 2011). As with the COMT Val genotype, a less focused activation pattern during working memory and episodic memory is often observed in older compared to younger adults (e.g., Bäckman et al., 1997; Dennis \& Cabeza, 2011; Grady, McIntosh, \& Craik, 2005; Logan, Sanders, Snyder, Morris, \& Buckner, 2002; Park et al., 2012; Rajah \& D'Esposito, 2005). Thus, we expected that the confluence of being older and being a COMT Val carrier would result in dedifferentiated brain activity during memory-related processing relative to younger adults, or to older adults carrying the Met variant. For example, in COMT Val carriers, aging may lead to an increased reliance of episodic memory processing on typical working memory structures (e.g., the fronto-parietal circuitry; Berryhill \& Olson, 2008; Cabeza, Dolcos, Graham, \& Nyberg, 2002; Naghavi \& Nyberg, 2005) and, conversely, working memory may be more dependent on typical episodic memory structures (e.g., the medial temporal lobe; Axmacher, Elger, \& Fell, 2009; Ezzyat \& Olson, 2008; Squire, Stark, \& Clark, 2004). In line with this assertion, a functional magnetic resonance study reported that normal aging increases the dependence of these two memory functions on common networks, with older adults showing increased recruitment of brain regions within a prefrontal-parietal-occipital network during both working and episodic memory (Sambataro et al., 2012). Moreover, working memory and episodic memory may be more dedifferentiated in older adults whose genetic predisposition for prefrontal DA modulation is suboptimal.

At the behavioral level, dedifferentiation also denotes increases in the correlations or dependence between various cognitive processes or abilities, such as different aspects of intelligence (e.g., Balinsky, 1941; Baltes, Cornelius, Spiro, Nesselroade, \& Willis, 1980; de Frias et al., 2007; Ghisletta \& Lindenberger, 2005; Li et al., 2004; Lindenberger \& Ghisletta, 2009; Tucker-Drob, 2009; Schaie, Maitland, Willis, \& Intrieri, 1998; but see Anstey, Hofer, \& Luszcz, 2003; Zelinski \& Lewis, 2003) or between sensory and cognitive processes (Baltes \& Lindenberger, 1997; Lindenberger \& Ghisletta, 2009; Lindenberger \& Baltes, 1994). However, it should be noted that longitudinal evidence suggests stronger dedifferentiation among processes in the cognitive domains compared to cross-domain cognitive-sensory dedifferentiation (Lindenberger \& Ghisletta, 2009).

Over a decade ago, a neurocomputational theory of cognitive aging suggested that increased processing noise due to suboptimal DA modulation results in increased within-network coactivation of distinct processing pathways in simulated older networks (Li \& Sikström, 2002) and higher correlations between the networks' performance tested with different memory tasks (Li, Lindenberger, \& Sikström, 2001).

Given the well-described effects of the COMT gene on working and episodic memory (for a review, see Witte \& Flöel, 2012), in this study we focus specifically on behavioral dedifferentiation of these two types of memory in old age to investigate whether aging-related memory dedifferentiation is related to individual differences in genetic predispositions for suboptimal prefrontal DA signaling. We investigated these questions in large samples of younger $(n=973)$ and older $(n=1,333)$ adults by comparing the latent structure, correlations, and means between a spatial working memory factor and a verbal episodic memory factor across COMT genotypes. Of special interest was the question whether increasing age is associated with increased correlations between working memory and episodic memory in the suboptimal COMT genotype. In line with the resource modulation hypothesis, which states that genetic variability is more likely to result in performance differences when brain resources are more limited, as in old age (Lindenberger, Nagel, Chicherio, Li, Heekeren, \& Bäckman, 2008), we expected to find the highest correlation between episodic memory and working memory in older COMT Val homozygotes, that is, in older adults carrying the genetic variant associated with the lowest level of prefrontal DA. 


\section{Methods}

\section{Participants}

We recruited 1,051 younger (20 to 31 years; $54.2 \%$ female) and 1,657 older (59 to 71 years; $61.0 \%$ female) adults via newspaper announcements and advertisements in public transportations. All subjects reported normal or corrected to normal vision and were right-handed, as indexed by the Edinburgh Handedness Index (Oldfield, 1971). Written informed consent was obtained from all participants, who were paid 10 Euro per hour for their participation. The ethics committees of the Charité University Medicine Berlin approved the study. Older participants that scored below 28 on the Mini-Mental State Examination were excluded from the sample (4.9\%), as scores below 28 may indicate mild cognitive impairment in highly educated elderly samples (O'Bryant et al., 2008). Furthermore, participants with a history of medical (e.g., heart attack), neurological (e.g., epilepsy), or psychiatric disease (e.g., depression) were excluded (younger adults: 6.3\%; older adults: $10.2 \%$ ), as well as participants taking drugs that may affect dopaminergic neuromodulation (younger adults: $1 \%$; older adults: $3.8 \%$ ). Finally, participants that had received less than 8 years of education were excluded ( $<1 \%$ for both age groups). The final sample included 973 younger (53.4\% female) and 1,333 older (60.4\% female) adults. The COMT genotypes did not differ with respect to the percentage of excluded participants within age groups (all $p \mathrm{~s}>.05$ for all exclusion criteria). Table 1 presents demographics and self-reported health data across age and COMT genotypes. Notably, the COMT groups did not differ with respect to demographic and self-reported health data in either age group. Althoug parts of the data have been published elsewhere (Li et al., 2010; Nagel et al., 2008), here we present data from a considerably larger sample and address a question that has not been investigate so far, namely the relationship between suboptimal DA modulation and cognitive dedifferentiation.

\section{Genotyping}

DNA was extracted from peripheral blood using standard methods. The COMT single nucleotide polymorphism (SNP, rs4680; Val158/108Met) was genotyped using the commercially available TaqMan 5' nuclease assay (C_25746809_50; TaqMan SNP genotyping Assay; Applied Biosystems, Forster City, CA), following established procedures (Livak, 1999). Genotyping was performed on 384-well microtiter plates in 5- $\mu$ l reaction volumes. For each reaction we combined 10 ng DNA template, $5 \times$ TaqMan genotyping assay and $5 \times$ TaqMan Genotyping Master Mix. Thermal cycling was done on a PTC-240 PCR instrument using the following cycling conditions: preamplification phase at $50^{\circ} \mathrm{C}(2 \mathrm{~min})$, initial denaturation at $95^{\circ} \mathrm{C}(10 \mathrm{~min})$, followed by 45 cycles of denaturation at $95^{\circ} \mathrm{C}(15 \mathrm{~s})$, annealing and extension at $60{ }^{\circ} \mathrm{C}(60$ $\mathrm{s})$. The genotype frequencies in the younger adults were 263 (27.0\%) for Met/Met, 504 (51.8\%) for Met/Val, and $206(21.2 \%)$ for $\mathrm{Val} / \mathrm{Val}$. The corresponding distributions for the older sample were 368 (27.6\%) for Met/Met, 680 for Met/Val (51.0\%), and 285 (21.4\%) for $\mathrm{Val} / \mathrm{Val}$. In both age groups, the COMT distributions were in Hardy-Weinberg equilibrium $(p s>0.1)$ and in line with the distributions reported in previous studies (e.g., Starr, Fox, Harris, Deary, \& Whalley, 2007).

\section{Spatial Working Memory Task}

The task used to assess spatial working memory has been described in detail by Nagel and colleagues (2008). Briefly, dots were presented one at a time in a specific location in a $4 \times 4$ grid. After a sequence of seven dots was presented, a probe appeared in one of the 16 locations. Participants were required to determine whether a dot was presented in this specific location (i.e., location memory condition). If the participant gave a yes response, a digit was presented in this location to probe participants to indicate whether the digit matched the serial position of the dot in the presented series (i.e., sequence memory condition). A total of 48 trials were presented. We used location memory accuracy and sequence memory accuracy to represent spatial working memory in latent space.

\section{Verbal Episodic Memory Task}

The task used to assess episodic serial order memory was the same as described by Li and colleagues (2010). Participants were asked to memorize three lists of 12 words each presented via headphones. Although the participants listened to the words, they simultaneously saw numbers on the computer screen, which represented the serial positions of the words. After list presentation, subjects recalled the items in backward order, beginning with the last item presented (i.e., Item 12 to Item 1). Given that the recency portion of backward serial recall relies more on short-term memory (Richardson, 2007), for later analysis only two indicator vari-

Table 1

Demographic Variables and Self-Reported Health Across Age and COMT Genotype Groups

\begin{tabular}{|c|c|c|c|c|c|c|}
\hline \multirow[b]{2}{*}{ Demographics } & \multicolumn{3}{|c|}{ Younger adults $(n=973)$} & \multicolumn{3}{|c|}{ Older adults $(n=1333)$} \\
\hline & $\begin{array}{c}\text { Met/Met } \\
(n=263)\end{array}$ & $\begin{array}{c}\text { Met/Val } \\
(n=504)\end{array}$ & $\begin{array}{c}\mathrm{Val} / \mathrm{Val} \\
(n=206)\end{array}$ & $\begin{array}{c}\text { Met/Met } \\
(n=368)\end{array}$ & $\begin{array}{c}\text { Met/Val } \\
(n=680)\end{array}$ & $\begin{array}{c}\mathrm{Val} / \mathrm{Val} \\
(n=285)\end{array}$ \\
\hline Age $(M \pm S D)$ & $26.4(2.8)$ & $25.9(2.8)$ & $25.9(2.7)^{\mathrm{a}}$ & $65.3(2.8)$ & $65.1(2.8)$ & $64.9(3.0)^{\mathrm{a}}$ \\
\hline$\%$ female & $53.2 \%$ & $53.0 \%$ & $54.9 \%^{\mathrm{b}}$ & $58.7 \%$ & $61.6 \%$ & $59.6 \%^{\mathrm{b}}$ \\
\hline Years of education $(M \pm S D)$ & $12.7(1.1)$ & $12.6(1.2)$ & $12.6(1.1)^{\mathrm{a}}$ & $10.7(1.7)$ & $10.8(1.7)$ & $10.9(1.7)^{\mathrm{a}}$ \\
\hline State of health $(M \pm S D)$ & $4.2(0.6)$ & $4.2(0.6)$ & $4.2(0.7)^{\mathrm{a}}$ & $3.9(0.7)$ & $4.0(0.6)$ & $4.0(0.6)^{\mathrm{a}}$ \\
\hline $\operatorname{MMSE}(M \pm S D)$ & & & & $29.23(0.8)$ & $29.39(0.7)$ & $29.42(0.7)^{\mathrm{a}}$ \\
\hline
\end{tabular}

Note. State of health is based on the mean of four self-ratings on 5-point scales from 1 (poor) to 5 (excellent). COMT = Catechol-O-Methyltransferase; MMSE = Mini-Mental State Examination.

${ }^{\mathrm{a}}$ One-way analyses of variance: n.s. ${ }^{\mathrm{b}} \mathrm{\chi}^{2}=$ n.s. 
ables were used to form the latent episodic memory factor: backward recall accuracy for the primacy (Items 1-4) and middle (Items 5-8) portions of the lists.

\section{Statistical Analysis}

To test whether the COMT gene affects memory dedifferentiation at the level of latent factor correlations, invariance of measurement models between COMT genotypes was evaluated by means of multiple group confirmatory factor analyses using AMOS 7.0 (Arbuckle, 2006; Byrne, 2004). A series of progressively more stringent models was evaluated, constraining different aspects of the measurement models (factor loadings, intercepts, residual variances, and interfactor correlations) to be equal across COMT genotype groups. Because the more constrained models were nested within the reference model, the difference in $\chi^{2}$ fit statistics $\left(\Delta \chi^{2}\right)$ was used to determine whether the models with more constraints yielded relatively better fits given the fewer number of parameters involved. The alpha-level for statistical decisions regarding differences in $\chi^{2}$ fit statistics was set to 0.05 . Gender and chronological age were included as covariates in all analyses. The proportion of missing data was rather minimal. In total, about $1.9 \%$ of the trials for the working memory task and less than $1 \%$ of the trials for the episodic memory task yielded missing data due to nonresponse of the participants.

Both univariate and multivariate outliers were examined. Specifically, cases exceeding \pm 3.29 SD (Tabachnick \& Fidell, 2005) were treated as univariate outliers and multivariate outliers were determined using Mahalanobis distance, with the recommended $p<.001$ threshold for the $\chi^{2}$ value (Tabachnick \& Fidell, 2005). In total, less than $1 \%$ of memory accuracy measures were deemed as outliers and were not included in further analyses (i.e., treated as missing by the program). AMOS accommodates analyses based on full information maximum likelihood estimation (FIML), assuming that data values that are missing are missing at random (Arbuckle, 1996). Older adults had more missing data on sequence memory accuracy in the working memory task, $\chi^{2}=(1,2306)=$ $38.8, p<.01$, indicating that they failed to respond in this condition more frequently than younger adults. Frequency of missing cases on the other indicators did not differ across age groups, $\chi^{2}=(1,2306)=n s$. Importantly, within age groups, the COMT groups did not differ with respect to missing values on any of the indicators (all $p \mathrm{~s}>.05$ ). For all following analyses, the raw variables were divided by an integer (i.e., 5) to avoid potential numeric estimation problems due to different scaling of indicators (cf. Kline, 2005).

\section{Results}

\section{The Effect of COMT Genotype on the Correlation Between Working Memory and Episodic Memory}

In the first step, a factor model was specified with two latent variables, each with two indicators. Figure 1 displays a graphical representation of the factor model. The reference model was identified by fixing the variances of the latent variables to 1 and constraining the factor loadings for each factor to be equal in each of the COMT genotype groups. Further analyses were conducted separately for younger and older adults, given that metric invari- ance could not be established across age groups, as indicated by a significant loss in fit when constraining the factor loadings to be equal across age groups, $\Delta \chi^{2}=37.46, \Delta d f=2, p<.05$. The lack of metric invariance between age groups is in line with evidence indicating life span age differences in the organization of cognitive abilities (e.g., Baltes et al., 1980; de Frias et al., 2007; Li et al., 2004; Tucker-Drob, 2009).

In the younger adults, the fit of the reference model (Model 1) was very good, indicating configural invariance in the three COMT groups, $\chi^{2}(21)=38.74, p<.05$, AIC $=158.7$, RMSEA $=$ $.030,90 \% \mathrm{CI}_{\mathrm{RMSEA}}(.014,044), \mathrm{CFI}=.98$ (all fit statistics are summarized in Table 2). The second, more constrained, model (Model 2) tested the hypothesis of identical magnitude of factor loadings across COMT genotypes; that is, metric (or weak) factorial invariance (Meredith, 1964). Model 2 also yielded very good fit, $\chi^{2}(25)=39.42, p<.05$, AIC $=151.4$, RMSEA $=.024,90 \%$ $\mathrm{CI}_{\text {RMSEA }}(.007,038), \mathrm{CFI}=.98$, and the restriction did not result in significantly worse fit compared to Model $1, \Delta \chi^{2}=0.68, \Delta d f=$ $4, p>.05$. Next, in addition to factor loadings, intercepts were constrained to be the same across COMT genotype groups (Model 3 ) to test for strong invariance. The fit of Model 3 remained very good, $\chi^{2}(33)=44.34, p>.05$, AIC $=140.3$, RMSEA $=.019$, $90 \% \mathrm{CI}_{\text {RMSEA }}(.000,032), \mathrm{CFI}=.99$. Subsequently, residual variances across genotype groups were equated (Model 4), establishing strict metric invariance, $\chi^{2}(41)=52.37, p>.05$, AIC $=$ 132.4 , RMSEA $=.017,90 \% \mathrm{CI}_{\mathrm{RMSEA}}(.000,029), \mathrm{CFI}=.99$ Finally, a model with equal interfactor correlations for the three COMT groups was tested, $\chi^{2}(43)=54.02, p>.05, \mathrm{AIC}=130.0$, RMSEA $=.016,90 \% \mathrm{CI}_{\text {RMSEA }}(.000,029), \mathrm{CFI}=.99$ (Model 5). Again the chi-square difference test was not significant, $\Delta \chi^{2}=$ $1.65, \Delta d f=2, p>.05$, indicating that the interfactor correlations could be equated across the three COMT genotype groups (i.e., $r s=.35, p<.05)$. Thus, strict metric invariance was achieved and the three interfactor correlations could be equated across the COMT genotype groups in younger adults. Factor loadings and interfactor correlations for the best fitting model in younger adults are displayed in Figure 1A.

The corresponding analysis in the older adults revealed very good fit of the reference model (Model 1), $\chi^{2}(21)=28.80, p>$ $.05, \mathrm{AIC}=148.8, \mathrm{RMSEA}=.017,90 \% \mathrm{CI}_{\mathrm{RMSEA}}(.000,031)$, $\mathrm{CFI}=.99$ (see Table 2 for all summary fit statistics). Constraining factor loadings (Model 2) across COMT genotypes to be equal did not significantly worsen the fit, $\Delta \chi^{2}=5.97, \Delta d f=4, p>.05$. Further, it was possible to achieve strong metric invariance (Model $3), \chi^{2}(33)=38.59, p>.05, \mathrm{AIC}=134.6, \mathrm{RMSEA}=.011,90 \%$ $\mathrm{CI}_{\text {RMSEA }}(.000,024), \mathrm{CFI}=.99$, as well as strict metric invariance (Model 4), $\chi^{2}(41)=51.50, p>.05$, AIC $=131.5$, RMSEA $=$ $.014,90 \% \mathrm{CI}_{\text {RMSEA }}(.000,025), \mathrm{CFI}=.98$. Importantly, however, the critical direct comparison of factor intercorrelations across older COMT genotype groups revealed that this constraint was associated with a significant decrement in model fit, $\Delta \chi^{2}=10.36$, $\Delta d f=2, p<.05$, indicating that factor intercorrelations differed significantly among the three COMT groups in older adults. The interfactor correlations in the heterozygotes and in Met homozygotes, however, could be equated without a significant loss in fit as compared to Model 4, $\Delta \chi^{2}=0.02, \Delta d f=1, p>.1$. Thus, Model 6 with strict metric invariance, but different factor correlations between Val homozygotes and the other two COMT groups, exhibited the best fit. In this model, the correlation between the 
A

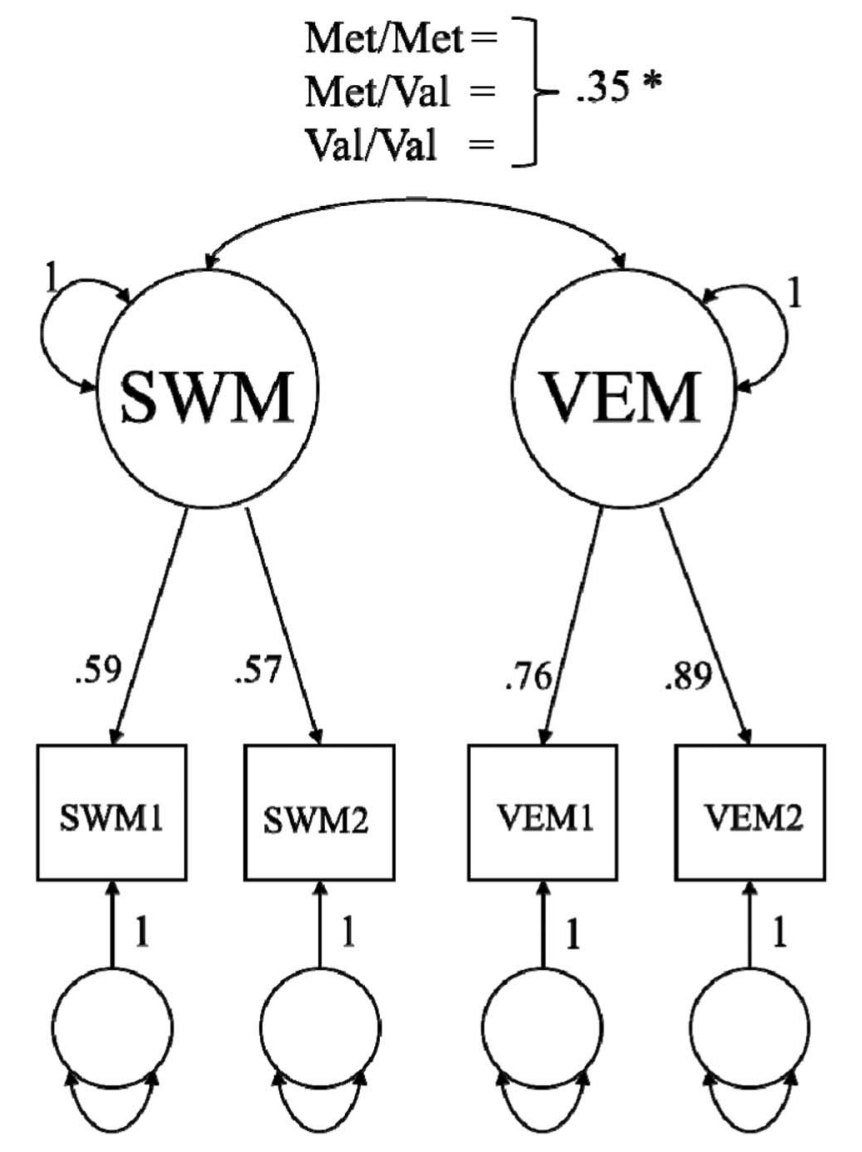

B

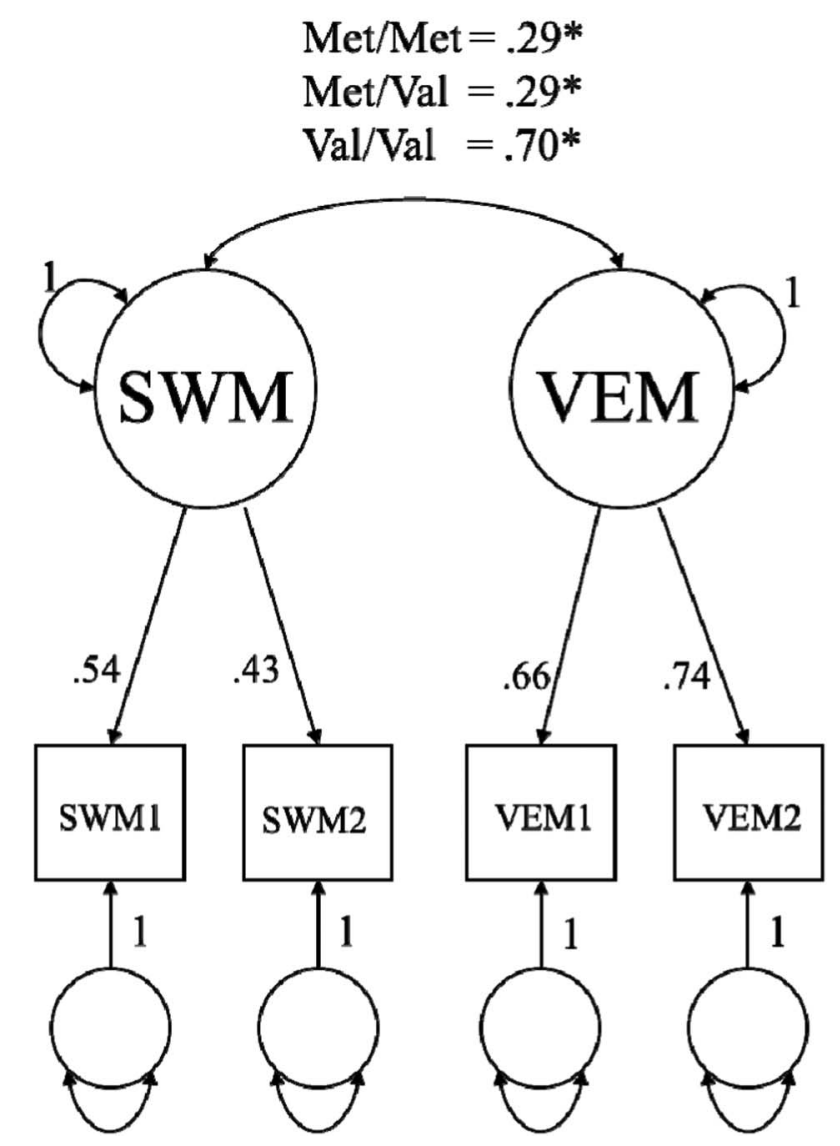

Figure 1. Factor model used in multiple group analyses on the relation between Catechol-O-Methyltransferase (COMT) genotype and dedifferentiation of memory functions. The figure depicts standardized factor loadings and interfactor correlations. A: Young adults (Met/Met: $n=263$; Met/Val: $n=504$; Val/Val: $n=206$ ): Shown here is the strict metric invariant model with equated interfactor correlations across all COMT genotypes. B: Older adults (Met/Met: $n=368$; Met/Val: $n=680$; Val/Val: $n=285$ ): Shown here is the strict metric invariant model with equated interfactor correlations across Met homozygotes and heterozygotes. Sex and chronological age are not shown in the figure but were included as covariates on the latent constructs in all analyses. SWM $=$ spatial working memory factor; VEM = verbal episodic memory factor; SWM1 $=$ SWM accuracy for the location memory condition; SWM2 = SWM accuracy for the sequence memory condition; VEM1 = backward recall for the primacy portion (Items 1-4); VEM2 = backward recall for the middle portion (Items 5-8) of the lists. ${ }^{*} p<.001$.

working memory and episodic memory factors was higher in older Val homozygotes $(r=.70, p<.001)$ compared to older heterozygotes and Met homozygotes $(r \mathrm{~s}=.29, p<.01)$. Figure $1 \mathrm{~B}$ displays the standardized factor loadings and the interfactor correlations of Model 6 for older adults.

In additional analyses, we tested whether the interfactor correlations could also be constrained to be equal across age groups. As before, the highest level of invariance that we could establish was strict metric invariance across genotype groups within each of the two age groups in a model involving all six groups, $\chi^{2}(80)=$ $103.87, p>.05, \mathrm{AIC}=263.4, \mathrm{RMSEA}=.011,90 \% \mathrm{CI}_{\mathrm{RMSEA}}$ $(.000,017), \mathrm{CFI}=.98$. Across age groups, configural invariance was achieved. In the next step, a model with equal interfactor correlations across all six COMT groups was tested. Equating the interfactor correlations across all six groups resulted in significant decrement in model fit, $\Delta \chi^{2}=12.05, \Delta d f=5, p<.05$, indicating that interfactor correlations differed significantly among the six groups. However, the interfactor correlations across all three younger COMT groups, the older heterozygotes, and the older Met homozygotes could be equated without significant loss in fit, $\Delta \chi^{2}=2.32, \Delta d f=4, p>.1$, compared to a model that imposed strict metric invariance within age groups and configural invariance across age groups. In line with the main results reported above, the correlation between the working memory and episodic 
Table 2

Fit Indices for Models Testing Different Types of Measurement Invariance

\begin{tabular}{|c|c|c|c|c|c|c|c|c|c|c|}
\hline Models & $x^{2}$ & $d f$ & $\chi^{2} / d f$ & AIC & RMSEA & $\begin{array}{l}90 \% \text { CI for } \\
\text { RMSEA }\end{array}$ & CFI & $\Delta \chi^{2}$ & $\Delta d f$ & $\Delta p$ \\
\hline \multicolumn{11}{|l|}{ Younger adults } \\
\hline Model 1: Configural invariance & 38.74 & 21 & 1.85 & 158.7 & .030 & {$[.014, .044]$} & .98 & - & - & - \\
\hline Model 2: Metric invariance & 39.42 & 25 & 1.58 & 151.4 & .024 & {$[.007, .038]$} & .98 & 0.68 & 4 & .954 \\
\hline Model 3: Strong invariance & 44.34 & 33 & 1.34 & 140.3 & .019 & {$[.000, .032]$} & .99 & 4.93 & 8 & .765 \\
\hline Model 4: Strict invariance & 52.37 & 41 & 1.28 & 132.4 & .017 & {$[.000, .029]$} & .99 & 8.02 & 8 & 431 \\
\hline $\begin{array}{l}\text { Model 5: Strict invariance and equal interfactor } \\
\text { correlation across all COMT groups }\end{array}$ & 54.02 & 43 & 1.26 & 130.0 & .016 & {$[.000, .029]$} & .99 & 1.65 & 2 & .439 \\
\hline \multicolumn{11}{|l|}{ Older adults } \\
\hline Model 1: Configural invariance & 28.80 & 21 & 1.37 & 148.8 & .017 & {$[.000, .031]$} & .99 & - & - & - \\
\hline Model 2: Metric invariance & 34.77 & 25 & 1.39 & 146.8 & .017 & {$[.000, .030]$} & .98 & 5.97 & 4 & .201 \\
\hline Model 3: Strong invariance & 38.59 & 33 & 1.17 & 134.6 & .011 & {$[.000, .024]$} & .99 & 3.82 & 8 & .873 \\
\hline Model 4: Strict invariance & 51.50 & 41 & 1.26 & 131.5 & .014 & {$[.000, .025]$} & .98 & 12.90 & 8 & .115 \\
\hline $\begin{array}{l}\text { Model 5: Strict invariance and equal interfactor } \\
\text { correlation across all COMT groups }\end{array}$ & 61.85 & 43 & 1.44 & 137.9 & .018 & {$[.006, .028]$} & .97 & 10.36 & 2 & .006 \\
\hline $\begin{array}{l}\text { Model 6: Strict invariance and equal interfactor } \\
\text { correlation across Met carriers }\end{array}$ & 51.51 & 42 & 1.23 & 129.5 & .013 & {$[.000, .024]$} & .98 & 0.02 & 1 & .890 \\
\hline
\end{tabular}

Note. $\quad \mathrm{AIC}=$ Akaike's information criterion; RMSEA $=$ root mean square error of approximation $\mathrm{CI}=$ confidence interval; $\mathrm{CFI}=\mathrm{comparative}$ fit index; COMT $=$ Catechol-O-Methyltransferase.

memory factors was higher in older Val homozygotes $(r=.71$, $p<.001)$ compared to the three younger COMT groups, older heterozygotes, and Met homozygotes $(r \mathrm{~s}=.33, p<.001)$. However, given that metric invariance did not hold across age groups, the results of these analyses need to be interpreted cautiously.

\section{Differences in Latent Means of Memory Factors Between COMT Groups}

In addition to testing differences in latent correlations, we investigated whether the COMT genotypes differed with respect to performance levels (i.e., latent means) in the two memory domains. Again, analyses were conducted separately for the two age groups. Given that strong invariance was established in both age groups (see above), latent mean differences could be compared across COMT groups. The latent means of the episodic memory and working memory factors were freed in the heterozygotes and Val homozygotes, and fixed to 0 in the Met homozygotes. In this way, the latent means are estimated as a relative difference from the reference point established by the Met homozygotes. In younger adults, the latent mean estimation for the best fitting model (Model 5) suggested that neither the heterozygotes nor the Val homozygotes differed reliably on verbal episodic memory (all $p$ s $>.1$ ) or spatial working memory (all $p \mathrm{~s}>.1$ ) from the Met homozygotes. The same was true when freeing the latent means in the groups of older heterozygotes and Val homozygotes for Model 6 , the best fitting model for the older age group. Thus, despite higher interfactor correlations in the group of older Val homozygotes, the latent means did not differ overall among COMT genotypes with respect to the two memory factors (all $p \mathrm{~s}>.1$ ).

\section{The Low Performing Older Adults From the Lower Tertile of the Distribution}

Although older Val homozygotes exhibited a higher link between memory functions, they did not differ in their latent means from the other two COMT genotype groups. However, task condition or genotype effects on mean performance levels may only be apparent at the low performance range (cf. Nagel et al., 2009; Papenberg et al., 2011). We, therefore, tested whether mean differences among older COMT genotypes would be apparent among the lower tertile of the distribution within each of the three allelic groups. Specifically, we expected that Val homozygotes in the lower tertile would perform worse than the other genotype groups on both memory measures.

Individuals in the lower tertile of the distribution (Met/Met: $n=$ 123; Met/Val: $n=226$; Val/Val: $n=94$ ) were selected based on an aggregate of the mean factor scores of episodic and working memory, which were computed from the best fitting model (i.e., Model 6 of strict metric invariance and equated factor correlations across Met homozygotes and heterozygotes). Bar graphs in Figure 2 portray factor scores on the two memory measures for the COMT genotype groups in the low-performing older adults. Univariate analyses of covariance, with sex and age as covariates, revealed main effects of COMT genotype with respect to both verbal episodic memory, $F(1,438)=3.7, p<.05$, partial eta squared $=.017$, and spatial working memory, $F(1,438)=4.6$, $p<.05$, partial eta squared $=.020$. In line with our expectations, planned contrasts indicated that Val homozygotes performed worse than heterozygotes and Met homozygotes with respect to both episodic memory, $t(438)=2.52, p<.05$, Cohen's $d=.24$, and working memory, $t(438)=3.02, p<.05$, Cohen's $d=.29$.

\section{Discussion}

We investigated whether suboptimal prefrontal DA modulation, associated with COMT Val homozygosity, affects the correlation between spatial working memory and verbal episodic memory. Our findings show that the latent correlations between the two memory factors was considerably higher in older Val homozygotes $(r=.70)$, compared to older heterozygotes and Met homozygotes ( $r$ s $=.29$ ). This finding was unique to the older adults. For 


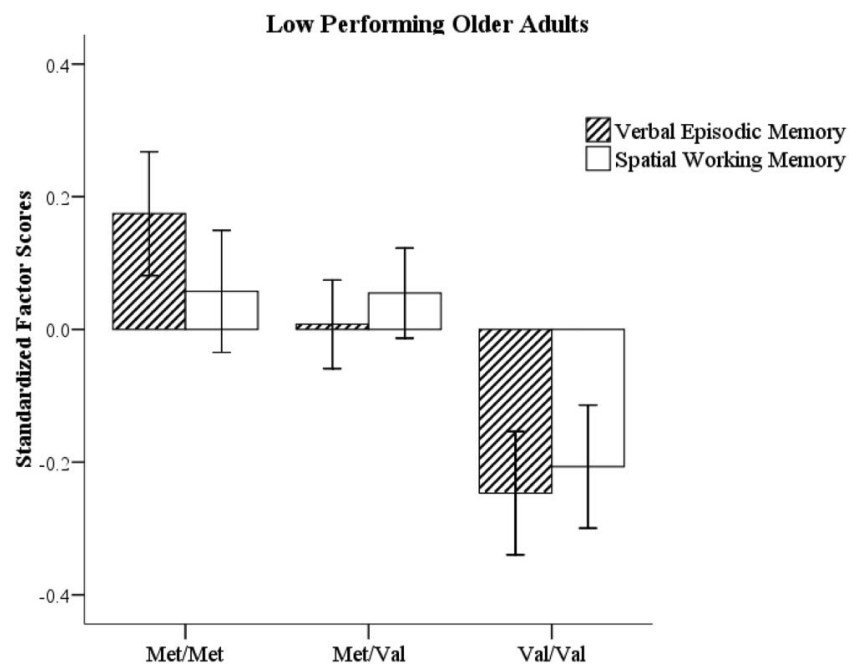

Figure 2. Bar graphs indicate standardized factor scores for verbal episodic and spatial working memory for the lower tertile of the distribution within each of the COMT groups (Met/Met: $n=123$; Met/Val: $n=226$; Val/Val: $n=94)$. Error bars represent 1 standard error around the mean.

younger adults, the relation between the two memory factors was invariant across the COMT genotypes $(r \mathrm{~s}=.35)$. The pattern of increased genetic effects on the coupling between working and episodic memory in old age is consistent with the resource modulation model, which assumes that genetic effects are magnified when cognitive resources decline, such as in aging (Lindenberger et al., 2008). In line with the predictions derived from earlier neurocomputational simulations (Li et al., 2001; Li \& Sikström, 2002), our findings thus lend empirical support for the theoretical conjecture postulating suboptimal DA modulation as one possible mechanism for the dedifferentiation of cognitive functions in old age.

Working memory and episodic memory have been shown to partly share common underlying neural networks (e.g., Cabeza et al., 2002; Ranganath, Johnson, \& D'Esposito, 2003). Our data also suggest that when frontal DA declines in late life (for review, see Bäckman et al., 2010), Val carriers' working memory may become increasingly dependent on other brain regions, such as those involved in episodic memory, and vice versa. This may result in higher correlations between memory functions at the behavioral level. Further, imaging studies have reported less focused brain activation patterns as well as recruitment of additional brain regions in older compared to younger adults (Grady et al., 2005; Rajah \& D'Esposito, 2005) and as a function of the COMT Val allele (for review, see Witte \& Flöel, 2012) during working memory and episodic memory. Our observation of less efficient and specific brain activations in Val carriers is in line with evidence showing that Val homozygotes are characterized by more noise in the neural signal and less distinctive brain activations during attentional processing (Winterer et al., 2006). Indeed, neurocomputational work has shown that simulated deficient DA modulation in networks with separate processing pathways for verbal and spatial memory leads to increased activation overlap across the two modules as a consequence of increased processing noise (Li \& Sikström, 2002). In addition to reduced functional specificity within networks, intercorrelations of different variants of memory tasks were higher in older networks with simulated suboptimal DA modulation (Li \& Lindenberger, 1999; Li et al., 2001). Thus, in line with the theory, our empirical evidence suggests that deficient DA modulation in older Val homozygotes may result in less specific neural processing during working memory and episodic memory, subsequently leading to an increased coupling between memory functions at the behavioral level.

In the total sample, COMT genotypes did not differ in latent means in either age group. Given previous reports of small or nonexistent effects of the COMT gene on mean performance of cognitive functions (see Barnett et al., 2007, 2008, for metaanalytic evidence), this result is not surprising. However, among the low-performing tertile of older adults, Val homozygotes performed less well in both memory functions than heterozygotes and Met homozygotes. This is also in line with the neurocompuational modeling results (Li \& Lindenberger, 1999; Li et al., 2001), which suggested that suboptimal DA modulation increases betweenperson heterogenity and lowers performance levels. Genotype effects on memory functions were not large enough to lower mean performance in the total sample of Val homozygotes below the mean of the heterozygotes and the Met homozygotes. However, when restricting the mean comparisons to the lower tertile of the distributions, we found that older Val homozygotes performed less well than the other two older groups on both cognitive abilities. In this sense, greater dedifferentiation of memory functions may serve as an early marker of future cognitive decline in older Val carriers. With advancing aging-related structural (e.g., Raz et al., 2005, 2008) and neuromodulatory (for review, see Bäckman et al., 2010) declines, genotype differences in performance levels may become apparent in the total sample. Indeed, longitudinal imaging studies have found that older adults with more diffuse brain activation patterns declined more in their clinical and neurological status, although these individuals did not differ in baseline memory performance from those with more distinct activation patterns (Bookheimer et al., 2000; O'Brien et al., 2010). Cross-sectional studies with even older samples and longitudinal studies are needed to confirm whether deficient DA modulation and its link to dedifferentiation result in worse performance outcomes with advancing adult age.

In this study, the candidate-gene approach served as a proxy for investigating the effects of individual differences in dopaminergic neuromodulation on adult age differences in the association between working and episodic memory. It would be desirable to complement our findings with molecular imaging studies, which permit a more direct assessment of individual differences in DA signaling. That said, a recent molecular imaging study in Parkinson's disease patients reported that Met exhibit higher DA levels in frontal regions than Val homozygotes (Wu et al., 2012).

In sum, we document novel evidence for an association between deficient frontal DA neuromodulation and aging-related dedifferentiation of memory functions at the behavioral level. Specifically, the correlation between working memory and episodic memory was higher in older COMT Val homozygotes relative to heterozygotes and Met homozygotes; in contrast, the COMT gene did not affect the correlation of memory functions in younger adults, suggesting an age-related magnification of genetic effects (Lindenberger et al., 2008). Future studies should test whether suboptimal DA availability in old age is related to dedifferentiation 
among broader domains of cognitive functions. The DA systems have also been strongly implicated in executive functioning (e.g., Nagel et al., 2008; Sheldrick et al., 2008) and recently also in attentional modulation (Störmer et al., 2012). Thus, interindividual differences in DA modulation of these processes and the interactions to episodic (e.g., Buckner, 2004; Chun \& Johnson, 2011; Sun et al., 2005) and working memory (e.g., Fisk \& Sharp, 2003; Zanto, Rubens, Thangavel, \& Gazzaley, 2011) may lead to stronger aging-related increases in correlations among these cognitive processes as well.

\section{References}

Anstey, K. J., Hofer, S. M., \& Luszcz, M. A. (2003). Cross-sectional and longitudinal patterns of dedifferentiation in late-life cognitive and sensory function: The effects of age, ability, attrition, and occasion of measurement. Journal of Experimental Psychology: General, 132, 470487. doi:10.1037/0096-3445.132.3.470

Arbuckle, J. L. (1996). Full information estimation in the presence of incomplete data. In G. A. Marcoulides, R. E. Schumacker (Eds.), Advanced structural equation modeling: Issues and techniques (pp. 243277). Mahwah, NJ: Erlbaum.

Axmacher, N., Elger, C. E., \& Fell, J. (2009). Working memory-related hippocampal deactivation interferes with long-term memory formation. The Journal of Neuroscience, 29, 1052-1960. doi:10.1523/JNEUROSCI .5277-08.2009

Bäckman, L., Almkvist, O., Andersson, L., Nordberg, A., Winblad, B., Reineck, R., \& Långström, B. (1997). Brain activation in young and older adults during implicit and explicit retrieval. Journal of Cognitive Neuroscience, 9, 378-391. doi:10.1162/jocn.1997.9.3.378

Bäckman, L., Lindenberger, U., Li, S.-C., \& Nyberg, L. (2010). Linking cognitive aging to alterations in dopamine neurotransmitter functioning: Recent data and future avenues. Neuroscience \& Biobehavioral Reviews, 34, 670-677. doi:10.1016/j.neubiorev.2009.12.008

Balinsky, B. (1941). An analysis of the mental factors of various age groups from nine to sixty. Genetic Psychology Monographs, 23, 191234.

Baltes, P. B., Cornelius, S. W., Spiro, A., Nesselroade, J. R., \& Willis, S. L. (1980). Integration versus differentiation of fluid/crystallized intelligence in old age. Developmental Psychology, 16, 625-635. doi:10.1037/ 0012-1649.16.6.625

Baltes, P. B., \& Lindenberger, U. (1997). Emergence of a powerful connection between sensory and cognitive functions across the adult life span: A new window to the study of cognitive aging? Psychology and Aging, 12, 12-21. doi:10.1037//0882-7974.12.1.12

Barnett, J. H., Jones, P. B., Robbins, T. W., \& Müller, U. (2007). Effects of the catechol-O-methyltransferase Val158Met polymorphism on executive function: A meta-analysis of the Wisconsin Card Sort Test in schizophrenia and healthy controls. Molecular Psychiatry, 12, 502-509. doi:10.1038/sj.mp.4001973

Barnett, J. H., Scoriels, L., \& Munafò, M. R. (2008). Meta-analysis of the cognitive effects of the catechol-O-methyltransferase gene Val158/ 108Met polymorphism. Biological Psychiatry, 64, 137-144. doi: 10.1016/j.biopsych.2008.01.005

Berryhill, M. E., \& Olson, I. R. (2008). The right parietal lobe is critical for visual working memory. Neuropsychologia, 46, 1767-1774. doi: 10.1016/j.neuropsychologia.2008.01.009

Bertolino, A., Rubino, V., Sambataro, F., Blasi, G., Latorre, V., Fazio, L., . . . Scarabino, T. (2006). Prefrontal-hippocampal coupling during memory processing is modulated by COMT val158met genotype. Biological Psychiatry, 60, 1250-1258. doi:10.1016/j.biopsych.2006.03.078

Blanchard, M. M., Chamberlain, S. R., Roiser, J., Robbins, T. W., \& Müller, U. (2011). Effects of two dopamine-modulating genes (DAT1 9/10 and COMT Val/Met) on n-back working memory performance in healthy volunteers. Psychological Medicine, 41, 611-618. doi:10.1017/ S003329171000098X

Bolton, J. L., Marioni, R. E., Deary, I. J., Harris, S. E., Stewart, M. C., Murray, G. D., . . Price, J. F. (2010). Association between polymorphisms of the dopamine receptor D2 and catechol-o-methyl transferase genes and cognitive function. Behavioral Genetics, 40, 630-638. doi 10.1007/s10519-010-9372-y

Bookheimer, S. Y., Strojwas, M. H., Cohen, M. S., Saunders, A. M., Pericak-Vance, M. A., Mazziotta, J. C., \& Small, G. W. (2000). Patterns of brain activation in people at risk for Alzheimer's disease. The New England Journal of Medicine, 343, 450-456. doi:10.1056/ NEJM200008173430701

Buckner, R. L. (2004). Memory and executive function in aging and AD: Multiple factors that cause decline and reserve factors that compensate Neuron, 44, 195-208. doi:10.1016/j.neuron.2004.09.006

Byrne, B. M. (2004). Testing for multigroup invariance using AMOS graphics: A road less traveled. Structural Equation Modeling: A Multidisciplinary Journal, 11, 272-300. doi:10.1207/s15328007sem1102_8

Cabeza, R., Dolcos, F., Graham, R., \& Nyberg, L. (2002). Similarities and differences in the neural correlates of episodic memory retrieval and working memory. Neuroimage, 16, 317-330. doi:10.1006/nimg.2002 .1063

Chun, M. M., \& Johnson, M. K. (2011). Memory: Enduring traces of perceptual and reflective attention. Neuron, 71, 520-535. doi:10.1016/ j.neuron.2011.10.026

Cools, R., \& D'Esposito, M. (2011). Inverted-u-shaped dopamine actions on human working memory and cognitive control. Biological Psychiatry, 69, e113-125. doi:10.1016/j.biopsych.2011.03.028

Deary, I. J., Penke, L., \& Johnson, W. (2010). The neuroscience of human intelligence differences. Nature Reviews Neuroscience, 11, 201-211. PMid:20145623.

de Frias, C. M., Lövdén, M., Lindenberger, U., \& Nilsson, L. G. (2007). Revisiting the dedifferentiation hypothesis with longitudinal multicohort data. Intelligence, 35, 381-392. doi:10.1016/j.intell.2006.07.011 de Frias, C., Marklund, P., Eriksson, E., Larsson, A., Öman, L., Annerbrink, K., . . . Nyberg, L. (2010). Influence of COMT gene polymorphism on fMRI-assessed sustained and transient activity during a working memory task. Journal of Cognitive Neuroscience, 22, 1614-1622. doi:10.1162/jocn.2009.21318

Dennis, N. A., \& Cabeza, R. (2011). Age-related dedifferentiation of learning systems: An fMRI study of implicit and explicit learning. Neurobiology of Aging, 32, e17-30. doi:10.1016/j.neurobiolaging.2010 .04 .004

Dennis, N. A., Need, A. C., LaBar, K. S., Waters-Metenier, S., Cirulli, E. T., Kragel, J., . . . Cabeza, R. (2010). COMT val108/158 met genotype affects neural but not cognitive processing in healthy individuals. Cerebral Cortex, 20, 672-683. doi:10.1093/cercor/bhp132

Dumontheil, I., Roggeman, C., Ziermans, T., Peyrard-Janvid, M., Matsson, H., Kere, J., \& Klingberg, T. (2011). Influence of the COMT genotype on working memory and brain activity changes during development Biological Psychiatry, 70, 222-229. doi:10.1016/j.biopsych.2011.02 .027

Egan, M. F., Goldberg, T. E., Kolachana, B. S., Callicott, J. H., Mazzanti, C. M., Straub, R., . . . Weinberger, D. R. (2001). Effect of COMT Val108/158 Met genotype on frontal lobe function and risk for schizophrenia. Proceedings of the National Academy of Sciences, USA, 98, 6917-6922. doi:10.1073/pnas.111134598

Ezzyat, Y., \& Olson, I. R. (2008). The medial temporal lobe and visual working memory: Comparisons across tasks, delays, and visual similarity. Cognitive Affective \& Behavioral Neuroscience, 8, 32-40. doi: 10.3758/CABN.8.1.32

Fisk, J. E., \& Sharp, C. A. (2003). The role of the executive system in visuo-spatial memory functioning. Brain and Cognition, 52, 364-381. doi:10.1016/S0278-2626(03)00183-0 
Ghisletta, P., \& Lindenberger, U. (2005). Exploring structural dynamics within and between sensory and intellectual functioning in old and very old age: Longitudinal evidence from the Berlin Aging Study. Intelligence, 33, 555-587. doi:10.1016/j.intell.2005.07.002

Grady, C. L., McIntosh, A. R., \& Craik, F. I. (2005). Task-related activity in prefrontal cortex and its relation to recognition memory performance in young and old adults. Neuropsychologia, 43, 1466-1481. doi: 10.1016/j.neuropsychologia.2004.12.016

Kimberg, D. Y., \& D'Esposito, M. (2003). Cognitive effects of the dopamine receptor agonist pergolide. Neuropsychologia, 41, 1020-1027. doi:10.1016/S0028-3932(02)00317-2

Kline, R. B. (2005). Principles and practice of structural equation modeling (2nd ed.). New York, NY: Guilford Press.

Li, S.-C., Chicherio, C., Nyberg, L., von Oertzen, T., Nagel, I. E., Papenberg, G., . . Bäckman, L. (2010). Ebbinghaus revisited: Influences of the BDNF Val66Met polymorphism on backward serial recall are modulated by human aging. Journal of Cognitive Neuroscience, 22, $2164-$ 2173. doi:10.1162/jocn.2009.21374

Li, S.-C., \& Lindenberger, U. (1999). Cross-level unification: A computational exploration of the link between deterioration of neurotransmitter systems and dedifferentiation of cognitive abilities in old age. In L.-G. Nilsson \& H. Markowitsch (Eds.), Cognitive neuroscience of memory (pp. 103-146). Seattle, WA: Hogrefe \& Huber.

Li, S.-C., Lindenberger, U., Hommel, B., Aschersleben, G., Prinz, W., \& Baltes, P. B. (2004). Transformations in the couplings among intellectual abilities and constituent cognitive processes across the life span. Psychological Science, 15, 155-163. doi:10.1111/j.0956-7976.2004 $.01503003 . \mathrm{x}$

Li, S.-C., Lindenberger, U., \& Sikström, S. (2001). Aging cognition: From neuromodulation to representation. Trends in Cognitive Sciences, 5, $479-486$

Li, S.-C., \& Sikstrom, S. (2002). Integrative neurocomputational perspectives on cognitive aging, neuromodulation, and representation. Neuroscience \& Biobehavioral Reviews, 26, 795-808.

Lindenberger, U., \& Baltes, P. B. (1994). Sensory functioning and intelligence in old age: A strong connection. Psychology and Aging, 9, 339-355. doi:10.1037//0882-7974.9.3.339

Lindenberger, U., \& Ghisletta, P. (2009). Cognitive and sensory declines in old age: Gauging the evidence for a common cause. Psychology and Aging, 24, 1-16. doi:10.1037/a0014986

Lindenberger, U., Nagel, I. E., Chicherio, C., Li, S.-C., Heekeren, H. R., \& Bäckman, L. (2008). Age-related decline in brain resources modulates genetic effects on cognitive functioning. Frontiers in Neuroscience, 2, 234-244. doi:10.3389/neuro.01.039.2008

Livak, K. J. (1999). Allelic discrimination using fluorogenic probes and the 5 ' nuclease assay. Genetic Analysis: Biomolecular Enginreering, 14, 143-149. PMid:10084106

Logan, J. M., Sanders, A. L., Snyder, A. Z., Morris, J. C., \& Buckner, R. L. (2002). Under-recruitment and non-selective recruitment: Dissociable neural mechanisms associated with aging. Neuron, 33, 827-840. doi: 10.1016/S0896-6273(02)00612-8

Lotta, T., Vidgren, J., Tilgmann, C., Ulmanen, I., Melen, K., Julkunen, I., \& Taskinen, J. (1995). Kinetics of human soluble and membrane-bound catechol O-methyltransferase: A revised mechanism and description of the thermolabile variant of the enzyme. Biochemistry, 34, 4202-4210. doi:10.1021/bi00013a008

Luciana, M., Depue, R. A., Arbisi, P., \& Leon, A. (1992). Facilitation of working memory in humans by a $\mathrm{d} 2$ dopamine receptor agonist. Journal of Cognitive Neuroscience, 4, 58-68. doi:10.1162/jocn.1992.4.1.58

Meredith, W. (1964). Notes on factorial invariance. Psychometrika, 29, 177-185. doi:10.1007/BF02289699

Nagel, I. E., Chicherio, C., Li, S.-C., von Oertzen, T., Sander, T., Villringer, A., . . L Lindenberger, U. (2008). Human aging magnifies genetic effects on executive functioning and working memory. Frontiers in Human Neuroscience, 2, 1. doi:10.3389/neuro.09.001.2008

Nagel, I. E., Preuschhof, C., Li, S.-C., Nyberg, L., Bäckman, L., Lindenberger, U., \& Heekeren, H. R. (2009). Performance level modulates adult age differences in brain activation during spatial working memory. Proceedings of the National Academy of Sciences, USA, 106, 2255222557. doi:10.1073/pnas.0908238106

Naghavi, H. R., \& Nyberg, L. (2005). Common fronto-parietal activity in attention, memory, and consciousness: Shared demands on integration? Conscious Cognition, 14, 390-425. doi:10.1016/j.concog.2004.10.003

O’Brien, J. L., O'Keefe, K. M., LaViolette, P. S., DeLuca, A. N., Blacker, D., Dickerson, B. C., \& Sperling, R. A. (2010). Longitudinal fMRI in elderly reveals loss of hippocampal activation with clinical decline. Neurology, 74, 1969-1976. doi:10.1212/WNL.0b013e3181e3966e

O'Bryant, S. E., Humphreys, J. D., Smith, G. E., Ivnik, R. J., GraffRadford, N. R., Petersen, R. C., \& Lucas, J. A. (2008). Detecting dementia with the Mini-Mental State Examination (MMSE) in highly educated individuals. Archives of Neurology, 65, 963-967. doi:10.1001/ archneur.65.7.963

Oldfield, R. C. (1971). The assessment and analysis of handedness: The Edinburgh inventory. Neuropsychologia, 9, 97-113. doi:10.1016/00283932(71)90067-4

Papenberg, G., Bäckman, L., Chicherio, C., Nagel, I. E., Heekeren, H. R., Lindenberger, U., \& Li, S.-C. (2011). Higher intraindividual variability is associated with more forgetting and dedifferentiated memory functions in old age. Neuropsychologia, 49, 1879-1888. doi:10.1016/j neuropsychologia.2011.03.013

Park, J., Carp, J., Kennedy, K. M., Rodrigue, K. M., Bischof, G. N., Huang, C.-M., . . . Park, D. C. (2012). Neural broadening or neural attenuation? Investigating age-related dedifferentiation in the face network in a large lifespan sample. The Journal of Neuroscience, 32, 2154-2158. doi: 10.1523/JNEUROSCI.4494-11.2012

Payton, A. (2009). The impact of genetic research on our understanding of normal cognitive ageing: 1995 to 2009. Neuropsychology Review, 19, 451-477. doi:10.1007/s11065-009-9116-z

Rajah, M. N., \& D'Esposito, M. (2005). Region-specific changes in prefrontal function with age: A review of PET and fMRI studies on working and episodic memory. Brain, 128, 1964-1983. doi:10.1093/brain/ awh608

Ranganath, C., Johnson, M. K., \& D’Esposito, M. (2003). Prefrontal activity associated with working memory and episodic long-term memory. Neuropsychologia, 41, 378-389. doi:10.1016/S00283932(02)00169-0

Rasch, B., Papassotiropoulos, A., \& de Quervain, D. F. (2010). Imaging genetics of cognitive functions: Focus on episodic memory. Neuroim age, 53, 870-877. doi:10.1016/j.neuroimage.2010.01.001

Raz, N., Lindenberger, U., Ghisletta, P., Rodrigue, K. M., Kennedy, K. M., \& Acker, J. D. (2008). Neuroanatomical correlates of fluid intelligence in healthy adults and persons with vascular risk factors. Cerebral Cortex, 18, 718-726. doi:10.1093/cercor/bhm108

Raz, N., Lindenberger, U., Rodrigue, K. M., Kennedy, K. M., Head, D., Williamson, A., . . Acker, J. D. (2005). Regional brain changes in aging healthy adults: General trends, individual differences and modifiers. Cerebral Cortex, 15, 1676-1689. doi:10.1093/cercor/bhi044

Richardson, J. T. (2007). Measures of short-term memory: A historical review. Cortex, 43, 635-650. doi:10.1016/S0010-9452(08)70493-3

Sambataro, F., Safrin, M., Lemaitre, H. S., Steele, S. U., Das, S. B., Callicott, J. H, . . . Mattay, V. S. (2012). Normal aging modulates prefrontoparietal networks underlying multiple memory processes. $E u$ ropean Journal of Neuroscience, 1-9. doi:10.1111/j.1460-9568.2012 .08254.x

Sawaguchi, T., \& Goldman-Rakic, P. S. (1991). D1 dopamine receptors in prefrontal cortex: Involvement in working memory. Science, 251, $947-$ 950. doi:10.1126/science.1825731 
Schaie, K. W., Maitland, S. B., Willis, S. L., \& Intrieri, R. C. (1998). Longitudinal invariance of adult psychometric ability factor structures across 7 years. Psychology and Aging, 13, 8-20. doi:10.1037/0882-7974 .13 .1 .8

Schott, B. H., Seidenbecher, C. I., Fenker, D. B., Lauer, C. J., Bunzeck, N., Bernstein, H.-G., . . . Düzel, E. (2006). The dopaminergic midbrain participates in human episodic memory formation: Evidence from genetic imaging. The Journal of Neuroscience, 26, 1407-1417. doi: 10.1523/JNEUROSCI.3463-05.2006

Sheldrick, A. J., Krug, A., Markov, V., Leube, D., Michel, T. M., Zerres, K., . . . Kircher, T. (2008). Effect of COMT val158met genotype on cognition and personality. European Psychiatry, 23, 385-389. doi: 10.1016/j.eurpsy.2008.05.002

Squire, L. R., Stark, C. E., \& Clark, R. E. (2004). The medial temporal lobe. Annual Review of Neuroscience, 27, 279-306. doi:10.1146/ annurev.neuro.27.070203.144130

Starr, J. M., Fox, H., Harris, S. E., Deary, I. J., \& Whalley, L. J. (2007). COMT genotype and cognitive ability: A longitudinal aging study. Neuroscience Letters, 421, 57-61. doi:10.1016/j.neulet.2007.05.023

Störmer, V. S., Passow, S., Biesenack, J., \& Li, S.-C. (2012). Dopaminergic and cholinergic modulations of visual-spatial attention and working memory: Insights from molecular genetic research and implications for adult cognitive development. Developmental Psycholology, 48, 875889. doi:10.1037/a0026198

Sun, X., Zhang, X., Chen, X., Zhang, P., Bao, M., Zhang, D., . . Hu, X. (2005). Age-dependent brain activation during forward and backward digit recall revealed by fMRI. Neuroimage, 26, 36-47. doi:10.1016/j .neuroimage.2005.01.022

Tabachnick, B. G., \& Fidell, L. S. (2005). Using multivariate statistics (5th ed.). New York, NY: Allyn \& Bacon.

Tucker-Drob, E. M. (2009). Differentiation of cognitive abilities across the life span. Developmental Psycholology, 45, 1097-1118. doi:10.1037/ a0015864

Vijayraghavan, S., Wang, M., Birnbaum, S. G., Williams, G. V., \& Arnsten, A. F. T. (2007). Inverted-U dopamine D1 receptor actions on prefrontal neurons engaged in working memory. Nature Neuroscience, 10, 376-384. doi:10.1038/nn1846

Williams, G. V., \& Goldman-Rakic, P. S. (1995). Modulation of memory fields by dopamine D1 receptors in prefrontal cortex. Nature, 376, 572-575. doi:10.1038/376572a0

Winterer, G., Musso, F., Vucurevic, G., Stoeter, P., Konrad, A., \& Seker, B., . . Weinberger, D. R. (2006). COMT genotype predicts BOLD signal and noise characteristics in prefrontal circuits. Neuroimage, 32, 1722-1732. doi:10.1016/j.neuroimage.2006.05.058

Witte, A. V., \& Flöel, A. (2012). Effects of COMT polymorphisms on brain function and behavior in health and disease. Brain Research Bulletin, 88, 418-428. doi:10.1016/j.brainresbull.2011.11.012

Wu, K., O'Keeffe, D., Politis, M., Robbins, G. C., Bose, S. K., Brooks, D. J., . . . Barker, R. A. (2012). The catechol-O-methyltransferase Val(158)Met polymorphism modulates fronto-cortical dopamine turnover in early Parkinson's disease: A PET study. Brain, 135, 2449-2457. doi:10.1093/brain/aws157

Zanto, T. P., Rubens, M. T., Thangavel, A., \& Gazzaley, A. (2011). Causal role of the prefrontal cortex in top-down modulation of visual processing and working memory. Nature Neuroscience, 14, 656-661. doi:10.1038/ nn.2773

Zelinski, E. M., \& Lewis, K. L. (2003). Adult age differences in multiple cognitive functions: Differentiation, dedifferentiation, or processspecific change? Psychology and Aging, 18, 727-745. doi:10.1037/ 0882-7974.18.4.727

Zilles, D., Meyer, J., Schneider-Axmann, T., Ekawardhani, S., Gruber, E., Falkai, P., \& Gruber, O. (2012). Genetic polymorphisms of 5-HTT and DAT but not COMT differentially affect verbal and visuospatial working memory functioning. European Archives of Psychiatry and Clinical Neuroscience. doi:10.1007/s00406-012-0312-0

Received July 29, 2012

Revision received January 9, 2013

Accepted March 6, 2013 